2003 Society for Design and Process Science

Printed in the United States of America

\title{
ENVIRONMENT IMPROVEMENTS FOR A BETTER COOPERATION IN MULTI-CULTURE COLLABORATIVE MECHANICAL DESIGN
}

\author{
Indira Thouvenin ${ }^{1}$, Marie-Hélène Abel ${ }^{2}$, Bruno Ramond $^{3}$ and Abir Qamhiyah ${ }^{4}$ \\ ${ }^{1}$ UTC, Universite de Technologie Compiègne,Roberval UMR 6066 - France \\ ${ }^{2}$ UTC, Université de Technologie de Compiègne, Heudiasyc UMR 6599 - France \\ ${ }^{3}$ UTC, Université de Technologie de Compiègne, Laboratoire CQP2 - France \\ ${ }^{4}$ Department of Mechanical Engineering, Iowa State University, USA
}

\begin{abstract}
In this paper a real example of a globally distributed collaborative design project is analyzed. Some of the problems identified as a result of this analysis include: 1- the lack of a complete design environment prior to the modeling stage, and 2- the need for a flexible and intuitive collaborative environment. To address these problems we propose some preliminary research results of task ontology, common to both cultures, in order to provide globally distributed design teams with a personal assistant agent.
\end{abstract}

Keywords: Computer aided design, collaborative and distributed design, task ontology, personal assistant agent

\section{Introduction}

Distributed collaborative design implies the ability of collaborating designers to work synchronously across geographic and time zones. For some years, a few experiments of nation-wide collaborative work have been conducted at the University of Technology of Compiegne (UTC) in France. In the Taxia project (Ramond, 1998), a hundred students from twelve engineering schools in France were assigned the prototype development of an industrial vehicle.

This paper examines closely an international collaborative design education experiment at the undergraduate level titled "CAD Across Universities (CADAU)" (Qamihiyah and Ramond, 2000). The experiment simulates a distributed and collaborative computer aided design project where students from the University of Technology of Compiegne (UTC) in France and Iowa State University (ISU) in the United States work together over the Internet on the design of a common product (Qamihiyah and Ramond, 1999). CADAU was developed by the mechanical engineering department at ISU in 1998 in collaboration with the International Institute for Theoretical and Applied Physics (IITAP) at ISU. International collaboration on CADAU between ISU and UTC began in September 1999. Section 2 describes the CADAU project.

A research project titled "Agent-Aided Cooperative Design" (AACC) has emerged at UTC based on the CADAU experiment to propose a more efficient collaborative design environment.. The goal of the study is to detect collaboration problems during the CADAU project, and to understand the reasons of success and failure in distributed and collaborative design.

Groupware or collaborative tools is one approach to be able to capture design intent, and quickly answer the user needs. But these tools cannot adapt themselves to the user. Another approach is to use 
a personal assistant agent. The agent has to work on a huge quantity of information such as mails, CAD files etc. In order to build it, we propose early stages of task ontology .

Preliminary results of the collaborative design tasks analysis are presented in Section 3 and Section 4 of this paper. Section 5 and Section 6 discuss the difficulties experienced by the students while using the modeling packages and the collaborative environment respectively. In Section 7 we discuss the differences in student's attitudes towards distance collaboration. The task ontology for the personal assistant agent is presented in Section 8. Section 9 and 10 give respectively the perspectives of this work and a conclusion.

\section{CADAU - a Framework for an Educational Multi-Culture Mechanical Design Project}

In CADAU French and American work together on a unified design project introduced with the CAD courses offered at ISU and UTC. Local student teams are formed at UTC and ISU. The UTC and ISU groups are then combined into international design teams. The goals of CAD courses (Yu et al., 1999) are the following:

1) To learn the theory and application of CAD tool. This is the most important scope of the course "CAD modeling initiation (TN20)" at the Mechanical Systems department at the University of Technology of Compiegne, and the course "Computer Aided Design (ME419)" at the Mechanical Engineering department, Iowa State University.

2) To give the students an international collaborative design experience with students in Mechanical Engineering from another country with a different language and a different culture.

To realize the second goal, a design project is proposed to groups composed of students from both countries. These groups have to design a product to satisfy predefined design specifications. Taking into account the limited time for the students to work on their project, three months of eight hours a week, the project doesn't include tolerance analysis and optimization of the design process.

At the beginning of the semester, the project is introduced simultaneously to the students by the two course instructors Dr Bruno Ramond (UTC) and Dr Abir Qamhiyah (ISU). At the first contact hour a project schedule, Table 1, is proposed to the students. French and American teams have to provide a common report at the end of the project.

Table 1: Project Schedule.

\begin{tabular}{|l|l|}
\hline $10^{\text {th }}$ of September & Start of the new term \\
\hline $16^{\text {th }}$ of October & Tasks list and market study \\
\hline $30^{\text {th }}$ of October & Design configuration \\
\hline $13^{\text {th }}$ of November & Modeling $\left(1^{\text {st }}\right.$ stage $)$ \\
\hline $27^{\text {th }}$ of November & Modeling $\left(2^{\text {nd }}\right.$ and final stage $)$ \\
\hline $11^{\text {th }}$ of December & Final report \\
\hline
\end{tabular}

The course includes lectures and computer laboratories. The first computer laboratory is devoted to the presentation of the design specifications. These specifications are defined by the French and American supervisors. The product is selected to be an innovative answer to everyday life needs such as a remotely controlled vacuum cleaner or a shopping cart with an automatic stair climber, etc.

Pro/Engineer is the CAD tool chosen by both course instructors. The collaborative work space was Basic Support for Cooperative Work (BSCW) then Pro Collaborate which are the most common tools in this field. A list of the CAD packages and collaborative design tools used in CADAU are presented in Table 2. 
Table 2: CADAU tools.

\begin{tabular}{|l|l|l|}
\hline & CAD tool & Collaborative work tool \\
\hline 1999 & Pro/Engineer & BSCW \\
\hline 2000 & Pro/Engineer & BSCW \\
\hline 2001 & Pro/Engineer & Pro Collaborate \\
\hline
\end{tabular}

The documentation on CAD design for the students is available from different sources including web sites on CAD solutions [http//bscw.gmd.de/about.html], web pages in ISU, and UTC. No books or reports are provided concerning the project, but the students are provided Internet access.

\section{First Results of the Collaborative Design Tasks Analysis}

Data from the collaborative CADAU work of the years 2000 and 2002 fall semesters were used for our analysis. Common project reports for the American and French students, course information for TN20 and ME419, student questions and comments, and several discussions between partners of the AACC project.

Documents collected on the BSCW site are emails, image files, and CAD models. The e-mail messages show two major subjects of discussion: communication and design. During the first lab student teams exchange e-mail to present themselves for their remote team members. Very quickly, design proposals are made and model images are exchanged. But collaboration is unequal and sometimes obscure.

\subsection{Initial Contact and Skills Assessment Made by the Teams}

The students began to meet by email and introduced themselves: name, training and course level, design and CAD competencies. The teams are required by the course instructor to select a project leader. American and French teams explained their constraints and work habits according to their culture and their respective course outlines and grading schemes. For example, students gave information about their study calendar, their course load, as well as the available time for the CAD project.

During the initial contact stage the following information elements are identified:

1) An individual initial contact

2) The selection of a project leader for each local team

3) A personal estimation of CAD competencies by each student

4) An estimation of the available time by student and team

5) The exchange of email addresses

\subsection{Intercultural Collaboration and Early Design}

After the initial contact, the teams began to define their design configurations to meet the design specifications and constraints provided by the course instructors. At this stage, we are able to observe the "motivation" or "demotivation" for collaboration. Reactions to the collaborative workspace emerge: the choice to use it completely, partially or not at all. This is related to the difficulties experienced by the students with technical or cultural communication.

This early stage of discussion and imagination is very important but rarely captured. The sketches and e-mail exchanges observed by the supervisors are not stored within the collaborative design tool. The computer tools to help this aspect of the work do not exist and the students draw rough sketches on paper and scan these documents to send them to their teammates. 
After the detailed design specifications are defined, each team runs a market study, an analysis of existing products, and looks for supporting design documentation. After this step, they start proposing ideas for the new product to be developed.

Technical questions such as respect of standards, safety, length, size, weight, and appearance and price constraints are examined in order to build a common prototype. Discussions about $110 \mathrm{~V}$ or $220 \mathrm{~V}$ electrical standards in USA and France or the metric system, show that cultural impact is extremely important for the product definition. Table 3 summarizes our observations of product representation and use as related to the country culture.

Table 3: Multi-culture comparison on product representation.

\begin{tabular}{|l|l|l|}
\hline Product to be designed & In France & In USA \\
\hline Vacuum cleaner & $\begin{array}{l}\text { Has to be beautiful. The marketing } \\
\text { and style are very important, but } \\
\text { the price may be triple of the one } \\
\text { in USA } \\
\text { Fully new vacuum cleaner }\end{array}$ & $\begin{array}{l}\text { Has to be efficient } \\
\text { and low cost. } \\
\text { The appearance is } \\
\text { less important }\end{array}$ \\
Motorized carrier & $\begin{array}{l}\text { Ellows frequent shopping, but few } \\
\text { existing product. }\end{array}$ \\
\hline things carried. & $\begin{array}{l}\text { Allows a weekly } \\
\text { shopping. }\end{array}$ \\
Used by a walker. & Used by a driver. \\
\hline
\end{tabular}

\section{Impact of collaborative design stages on modeling}

After the first product sketches a phase of research and solution evaluation is observed, with discussions and the elimination of complex or nonadequate solutions. This situation lacks computer aid, knowledge management and information storage.

Next the final product is modeled. Students began to work on separate elements, with a CAD tool: this means that they had a very precise idea of what they wanted to represent at this time of the project. We can conclude that modeling needs a collaborative effort less important than preceding stages.

Taking into account the project context, project management is often lacking. Some students worked separately, and did not collaborate at all. Others worked together at the beginning then used the modeling tool in parallel to do the assembly at the end if possible. On the other hand, some groups collaborated closely following a properly defined plan of work. For such groups we observed a precise task definition and assignment in the team.

\section{Difficulties in modeling environment}

During computer laboratories the supervisors observed that students did not model easily, did not choose a good method which is understandable. Each team worked independently and sometimes wanted to force the CAD tool to do what they wanted, without taking into account the tool "logic".

Another point is that the students were not always conscious of the module they used in the modeling environment, and did not know how to access the one which interested them. CAD software has adopted a "windows look" where all the menus and windows may be adapted to the user. Every one can setup his or her own work space, which is very useful when he (or she) has a good knowledge of the tool, but may be very disturbing while training. The students need minimal and easy to access documentation on modeling, referring to the tool, and they need to identify in which module they need to work. 
Modeling is not supposed to be realized with a rigid methodology; it depends strongly on the CAD tool, and over all, on the users. It can be interesting to look for standard components, but the risk is that students do not completely model their project and only do product assembly.

Here, we notice that the collaborative aspect is not tremendously important. On the other hand, it would be interesting to capitalize problems and to offer documentation aid. Also, information related to industrial mechanical design projects is rarely available, and the students are not able to access them easily.

\section{Difficulties in collaboration environment}

The first problem is language: French students need help in English, and ask for email translation. On line translation with Alta vista or other sites is not satisfying, which leads to a poor understanding of received and sent mails by French students, and obscure writing. American students do not usually learn foreign languages.

Difference in time zones is surprisingly not a problem: messages have to be read, translated and understood, and this takes time. For the low level at English for a French student, it seems that an asynchronous communication is better for collaboration. This is something important in a real collaborative CAD project, even if the idea of a synchronous work is attractive.

The second problem is that of the academic calendar which is different between France and the United States. For example, during examination weeks, students have difficulties communicating: the lack of contact between teams induces a loss of motivation.

The collaborative work environment represents the third problem. Students do not use it for several reasons:

- They need training to use the tool, and they are lost with the online help.

- This tool is limited by the universities computer security (this is solved easily when the problem is identified). Students prefer to use their personal mail: a heavy loss for the collaborative work analysis is then generated due to emails dispersion. The collaborative tool is seen as unintuitive particularly for CAD file exchanges. Teams do not go to the collaborative space to read information on the CAD project.

- File management and workflow are not taken into account.

- American and French students do not have exactly the same training methods, and do not work in the same way. For example, during the semester, the CAD class and labs in USA are not exactly the same (number of hours) as the one in France. This is very interesting for them, if they find a good compromise for the working rhythm.

There is a more general problem in CAD project collaboration: the perception of the presence of the other team, and the impression that one or several human beings are at the other side of the ocean to work on the same project.

\section{Presence perception and inter cultural awareness in distance collaboration}

The main difference between a team which succeeds in collaborating and another which collaborates less seems, according to the course instructors, to be related to a common motivation, a presence perception and a cultural understanding between teams. The students have difficulties in working with persons they have never met physically.

- Best collaboration was performed by teams having an experience concerning the other country: French students who have spent one year exchange in USA, or American students who had taken courses of French language. Some of the students had taken a class about intercultural management.

- Some students had negative preconception of other cultures. 
Through this experience, it is easy to understand that common motivation and desire to collaborate are related. During the design configuration stage the cultural differences strongly influence the adopted solution.

Collaboration has to be clear and efficient from the beginning, and to perform it, it seems that presence feeling or "cyber presence" should be examined. This feeling is reinforced according to Mantovani and Riva (1999) when users work with the same task ontology, the one who are concerned by collaborative mechanical design in our case.

We have therefore analyzed more deeply these tasks in order to define common collaborative design task ontology to help users both for collaboration and mechanical design. To capture design intent, instant creation (Yu et al., 1999) and the teams shared ideas, we build a common space where an intelligent agent will be able to operate for knowledge management (Hoyle and Lueg, 1997) and for help while performing the project. In addition, this common space, related to a common ontology, facilitates a close collaboration: students feel more comfortable in a common familiar environment.

In Figure 1, we represent how the intelligent agent (Tacla and Enembreck, 2002) or the "personal assistant agent" is positioned between the user, the collaborative space and the public space.

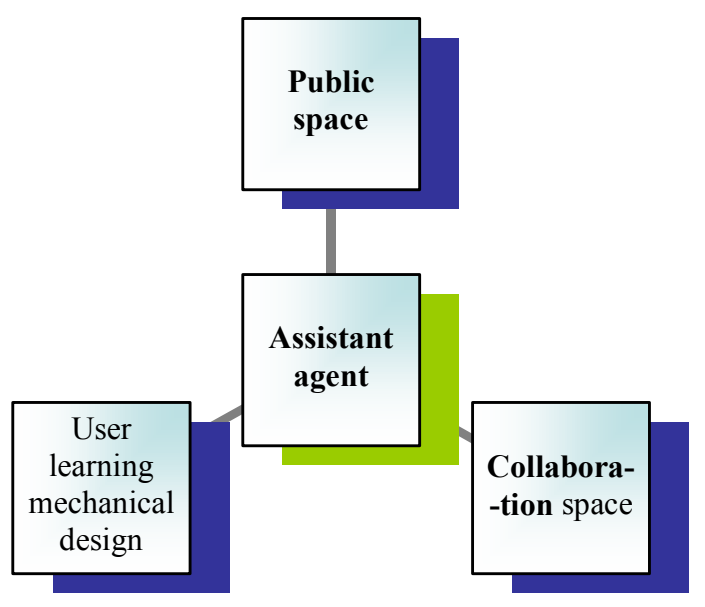

Figure 1:Assistant agent for collaborative design education.

The assistant agent is not supposed to act on the users work, but may offer them information they need just in time. For dimensioning, for example, the agent can propose previous projects reports by students with the same scope. It can also index documents to share and organize information. This part of the research project is presented by Enembreck and Barthès (AACC member) (2003).

\section{Task ontology}

In order to allow for the agent to do a task analysis during the design process, we have decided to define task ontology invisible for the user. The assistant agent is supposed to use this ontology to be able to act as described previously in (Hoyle and Lueg, 1997).

In fact, these tasks do not appear explicitly during the whole process. Choices are not clearly argued, or the discussion seems to be lost. As it is difficult to establish an ontology (Gruber, 1993) in these conditions, we separate the ontology related to modeling, and the one related to collaborative design without the modeling part. 
An ontology may be defined by a concept hierarchy, links between concepts and inference rules. Among links between tasks we find subsumption links ("is-a"), composition links ("part of"), or precedence links. Our work is focused on the training project dedicated ontology: we are not doing an absolute ontology. The students need help mainly for collaboration tasks and a little for modeling. In this paper, we will present a part of the built ontology. All the concepts have been defined according to documents such as mails, reports, or discussions with the teachers. But this work can be more generic and be used for other kind of collaborations.

\subsection{Ontology Related to a Modeling Tool}

For this project, students have used a popular CAD tool, Pro Engineer (PTC), in the two universities. This way, they adopted the corresponding domain ontology. This tool is widely explored and well implemented in the CAD community.

Some tasks like "extrusion" cannot be done if another task, like a "sketch" (2D drawing), is necessary before. Modeling tasks are clear, and managed by the user, with the CAD tool directly. Of course the related ontology doesn't represent the vocabulary and complexity in mechanical design. Nevertheless, we do not considered it useful to list all the terms used by Pro Eng: the CAD tool cannot be modified, and the modeling intent cannot be clearly seen by an assistant agent, even with task ontology for modeling. In addition, there are a lot of CAD tools using modeling and assembly ontology, and each tool is a strong guide for the user.

\subsection{Ontology Related to Mechanical Design Before the Modeling Stage}

After the project development analysis, we have collected implicit and explicit tasks.

Explicit tasks were detected with indications in mails like "we would like to build the vacuum cleaner with 12 volts continuous power battery to have a wireless system" or "it is probably easier to work with the International Measurement System (meter second kilogram)".

Concerning implicit tasks, we have imagined them for when a team proposes a solution where no problem is issued. For example, when a team writes "we have received your options and we both prefer the second one because ..." it is necessary to come back to the possible options referring to the answer.

We have defined and organized these tasks using an ontology (Gruber, 1993). This task ontology contains sub-ontologies. For example, among the collected tasks, some are related to consulting (Figure 2) between the teammates. Other tasks require a personal work as looking for ideas, or solutions or analysis.

Figure 2 illustrates an extract of the tasks sub-ontology where students have to consult themselves. The use of task ontology allows the agent to clear up affected tasks and to position them among the whole design process. 

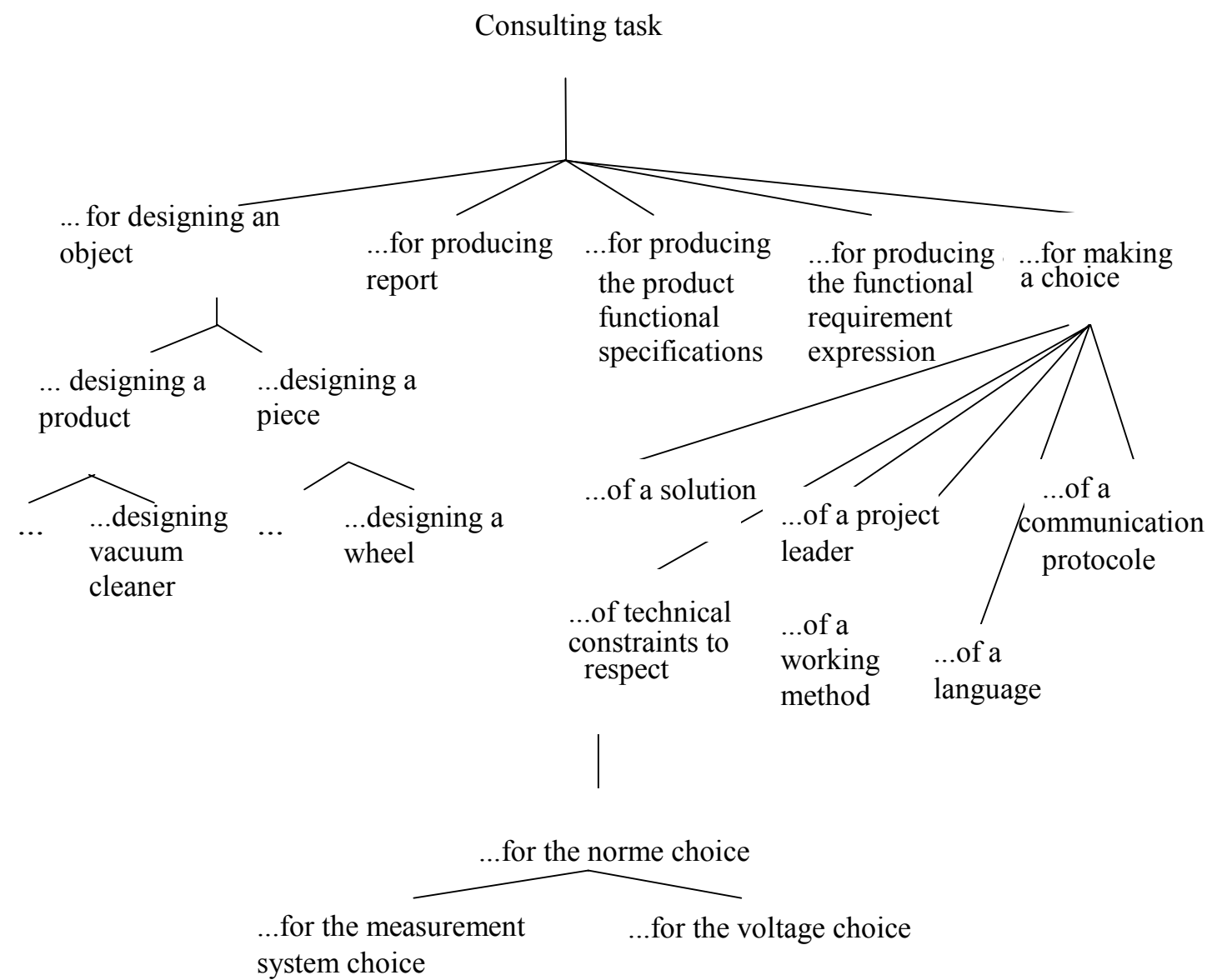

Figure 2: Part of the consulting tasks of the term hierarchy.

Based on the task ontology, for example, the agent will be able to detect a standard choice task and propose the students some World Wide Web sites on this subject. Or the agent will be able to detect a communication task and will memorize the exchanges related to this task.

This ontology will be completed and enhanced by new educational projects with other countries.

\subsection{Description and links between tasks}

First we present the observed main tasks, and then we describe links between tasks.

\subsubsection{Main tasks}

We have observed eight main tasks where students need help. These tasks are sub ontologies roots:

- a study task consists of studying, analyzing an object

- a presentation task consists of presenting information

- a handling task consists of apprehending an object

- a consulting task consists of consulting each other for a precise aim

- a drafting task consists of presenting written results

- an evaluation task consists of evaluating an object

- a research task consists of doing a research action 
- an understanding task consists of giving oneself the means to understand

\subsubsection{Links between tasks}

We used three kinds of links: subsumption, composition, and precedence.

\section{a) Subsumption links}

A subsumption link ("is-a") allows to refine tasks. For example, as in Figure 2, we see that a voltage choice is a norm choice task, which is a technical constraint choice to respect task, which is a choice task, which is a consulting task.

\section{b) Composition links}

A composition link (“part of") allows defining a task as a composition of different sub tasks.

The consulting task to choose the working method, for example, is composed of the language choice task and the communication protocol task as described in Figure 3.

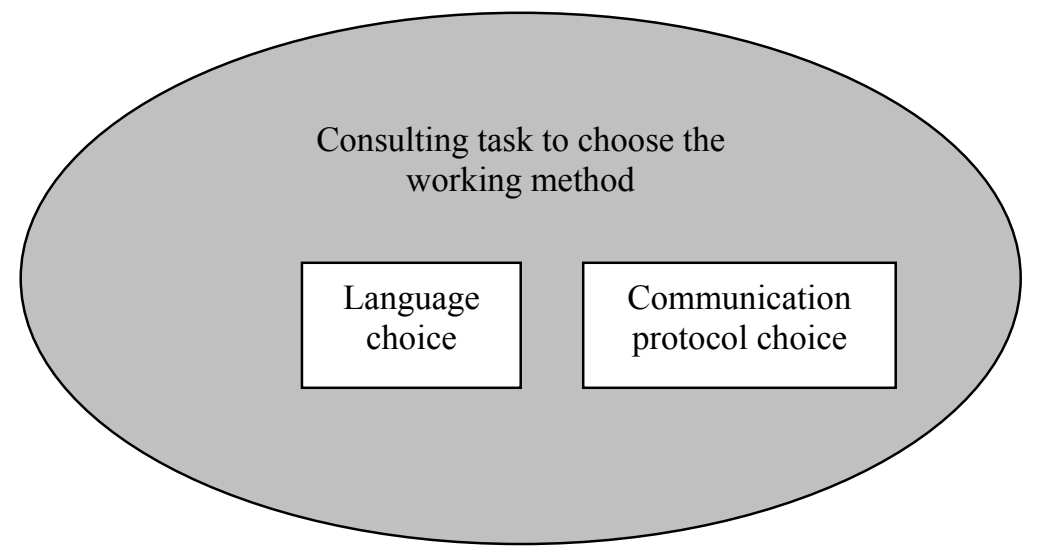

Figure 3: Composition link.

\section{c) Precedence links}

A precedence link defines the tasks that have to be executed before others. As an example, it is necessary to choose a project leader (Figure 4) before choosing the working method.

$\begin{array}{|lll|}\begin{array}{l}\text { Consulting task } \\ \text { to define the } \\ \text { functional } \\ \text { requirement }\end{array} & \text { precedes } & \text { Faisability study } \\ \text { task }\end{array}$

Figure 4: Precedence link.

As we can see in Figure 4, the feasibility study task is not a consulting task. The precedence links can operate between tasks from different subontologies.

We saw that the assistant agent should communicate with the user and capitalize the design process, detecting tasks or related domains tasks. But how will this run? A tentative of drawing a user interface is presented in the next part. 


\section{User interface perspectives}

We extracted four main functions during the collaborative design: communication, overview of the project including capitalization and management, documentation and dialog. In Table 4, the framework of this user interface is described.

Table 4: A user interface for assistant agent using task ontology

\begin{tabular}{|l|l|}
\hline $\begin{array}{l}\text { User work space } \\
\text { Emails, chat, applications used by the user }\end{array}$ & $\begin{array}{l}\text { Collaborative work space } \\
\text { Project information, management and } \\
\text { concerned partners }\end{array}$ \\
\hline $\begin{array}{l}\text { Information proposed by the agent place } \\
\text { Agent reactions: the agent detects tasks, offers } \\
\begin{array}{l}\text { some aid during the project, and capitalizes at } \\
\text { the same time }\end{array}\end{array}$ & $\begin{array}{l}\text { Dialog place } \\
\text { Questions -answers between the agent } \\
\text { and ther }\end{array}$ \\
\hline
\end{tabular}

Then Table 5 gives an example of the user and the agent reactions, on a specific problem of consulting.

Here the agent has detected that some students from UTC were consulting students from ISU to choose a working method. The ontology is invisible for the users. The agent detects a task which is implicit.

Table 5: Example of the assistant agent interventions and reactions during the design

\begin{tabular}{|l|l|}
\hline $\begin{array}{l}\text { User work space } \\
\text { "How are we going to work together for this project?" }\end{array}$ & Collaborative work space \\
\hline $\begin{array}{l}\text { Information proposed by the agent place } \\
\quad \begin{array}{l}\text { Define a project leader } \\
\text { Define a communication protocol }\end{array}\end{array}$ & Dialog place \\
\hline \begin{tabular}{l} 
Definguage (English/ French) \\
\hline
\end{tabular} & \\
\hline
\end{tabular}

Another way for the agent to offer some help is presented in Table 6:

a) to offer some documents in the collaborative work space

b) to answer to an explicit question, in the dialog place, or to let the students ask some questions 
Table 6: Example of the assistant agent documentation help and of the assistant agent and the user dialog

\begin{tabular}{|c|c|}
\hline User work space & $\begin{array}{ll}\text { Collaborative work space } \\
- & \text { Project Vaccuum cleaner design } \\
- & \text { Partners Amanda, W., Gregory, W., } \\
& \text { Thomas, H., Thomas, D. } \\
- & \text { Deadlines } \\
- & \text { Documents }\end{array}$ \\
\hline \multirow{3}{*}{$\begin{array}{l}\text { Information proposed } \\
\text { by the agent place }\end{array}$} & Dialog place \\
\hline & $\begin{array}{l}\text { I need some help for the working method } \\
\text { with American students. }\end{array}$ \\
\hline & $\begin{array}{l}\text { You can use the templates for each } \\
\text { student and try to follow some essential steps }\end{array}$ \\
\hline
\end{tabular}

This framework is a new idea and has to be implemented and experimented with the agent. This will be the next part of our work in order to get an efficient tool including the agent, ontologies and mechanical design documentation for a collaborative educational project.

\section{Conclusion}

In this paper, we analyzed CADAU, a distributed and collaborative experience in mechanical design between Iowa State University and University of Technology of Compiegne. The analysis is a research project (AACC) developed at UTC to understand and improve collaborative design environments. The conclusion of this analysis is that:

- The mechanical design environment is not complete, particularly in the early stages of the design process where exchanges, discussions, solution propositions are not capitalized or aided

- The collaborative environment is not fully exploited, and is not providing easy communication, intuitive access and documentation

- Intercultural awareness is a very important point: it means foreign language learning, but also a different view of new product according to the culture for appearance, functions, and standards.

- The adaptation effort for international collaboration may lead to a demotivation or a loss in the desire to collaborate, which has to be understood and anticipated.

We present some preliminary research results of task ontology, common to both cultures in order to provide a personal assistant agent to the user during the international collaborative design process. This work is in progress and we plan to complete it with future students projects. The assistant agent is also an ongoing work.

In a near future, this collaborative CAD class will be extended to Brazil and Romania, to reinforce the multicultural aspect of the collaboration, and we are considering a virtual environment development to collaborate intuitively. 


\section{Acknowledgments}

We acknowledge Dr Jean Paul Barthès and Fabrizio Enembreck, AACC members, and Rony Kubat who participates in TN20. This research project is supported by Region Picardie in France.

\section{References}

Ramond, B., 1998, “Collaborative Design in Education: the Taxia Project”, Proceedings of IDMME'98, pp 1261-1268, Compiègne, France.

Qamihiyah, A.Z., Ramond, B., 2000, "International Collaboration in mechanical Computer-Aided Design Education”, ASEE 2000.

Qamhiyah, A.Z., Ramond, B., 1999, "CAD Across Universities- An international Collaborative mechanical Engineering Education Experiment", Proceedings of the Fourth International Workshop on Computer Supported Collaborative Work in Design, Compiègne, France.

Yu, J., Shi, Y., Yang, H., Ramond, B., and Qamhiyah, A., 1999, "Collaborative Mechanical Engineering, Education Through Internet: CAD Across Universities".

Mantovani, G., Riva, G., 1999, "Real presence: How different ontologies generate different criteria for presence, telepresence, and virtual presence", Journal of presence, teleoperators and Virtual environments, 8(5), pp 538- 548.

Hoyle, M.A. and Lueg, C., 1997, "Open Sesame !: a Look at Personal Assistants", Proceedings of the International Conference on the Practical Application of Intelligent Agents and Multi-Agent Technology (PAAM 97), London, UK, pp 51-60.

Grüber, T.R., 1993, "A translation approach to portable ontology specifications", Knowledge Acquisition 5, p 199-220.

Tacla, C.A. and Enembreck, F., 2002, "Organizing R\&D experiences using agents", Starting Artificial Intelligence Researchers Symposium - STAIRS 2002, Lyon, France.

Enembreck, F. and Barthès, J.-P., 2003, "Improving Computer Supported Cooperative Design With Personal Assistant Agents", Journal of Integrated Design and Process Science, Vol. 7, No. 2, pp. 3-19. 